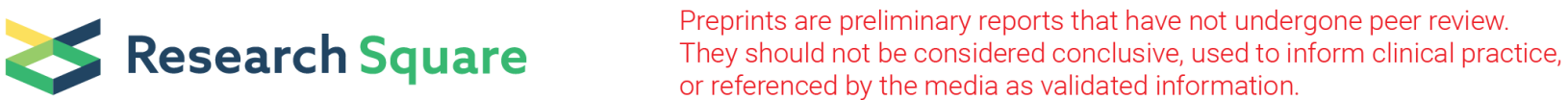 \\ Photoinduced regioselective olefination of arenes at proximal and distal sites
}

Debabrata Maiti ( $\sim$ dmaiti@iitb.ac.in )

Indian Institute of Technology (IIT) Bombay https://orcid.org/0000-0001-8353-1306

\section{Argha Saha}

IIT Bombay

\section{Srimanta Guin}

IIT Bombay

\section{Wajid Ali}

IIT Bombay

\section{Trisha Bhattacharya}

IIT Bombay

\section{Sheuli Sasmal}

IIT Bombay

\section{Nupur Goswami}

Indian Institute of Technology (IIT) Bombay

\section{Gaurav Prakash}

IIT Bombay

\section{Soumya Sinha}

Indian Institute of Technology (IIT) Bombay

Hediyala Chandrashekar

IIT Bombay

\section{S. S. Anjana}

IIT Bombay

\section{Physical Sciences - Article}

Keywords: Fujiwara-Moritani Reaction, C-H Activation Protocols, Reactivity, Regioselectivity, Olefination, Photoinduced Attainment

Posted Date: December 9th, 2020

DOl: https://doi.org/10.21203/rs.3.rs-118633/v1

License: (9) This work is licensed under a Creative Commons Attribution 4.0 International License. Read Full License 
Page $2 / 21$ 


\section{Abstract}

The Fujiwara-Moritani reaction has had a profound contribution in the emergence of contemporary $\mathrm{C}-\mathrm{H}$ activation protocols. Despite the applicability of the traditional approach in different fields, the associated reactivity and regioselectivity issues had rendered it redundant. The revival of this exemplary reaction requires the development of a mechanistic paradigm that would have simultaneous control on both the reactivity and regioselectivity. Often high thermal energy required to promote olefination leads to multiple site functionalization. To this aim we established a photoredoxcatalytic system constituting a merger of palladium/organo-photocatalyst that forges oxidative olefination in an explicit regioselective fashion of diverse arenes and heteroarenes. Visible light plays a significant role in executing 'regio-resolved' Fuijiwara-Moritani reaction without the requirement of silver salts and thermal energy. The catalytic system is also amenable towards proximal and distal olefination aided by respective directing groups (DGs), which entails the versatility of the protocol in engaging the entire spectrum of $\mathrm{C}(\mathrm{sp} 2)-\mathrm{H}$ olefination. Furthermore, streamlining the synthesis of natural products, chiral molecules, drugs and diversification through late-stage functionalizations underscore the importance of this sustainable protocol. The photoinduced attainment of this regioselective transformation is mechanistically established through control reactions, kinetic studies and theoretical calculations.

\section{Main Text}

The inception of Fujiwara-Moritani reaction had established a profound platform in the area of oxidative $\mathrm{C}-\mathrm{H}$ functionalizations (Fig. 1a) ${ }^{1}$. This exemplary reaction, although inherits the advantages of a $\mathrm{C}-\mathrm{H}$ activation process, falls apart in regioselectivity and reactivity ${ }^{2}$. Very recently ligand approaches have been devised which helped in overcoming the reactivity issue and expanded the scope to various arenes and heteroarenes (Fig. 1b) ${ }^{3,4}$. However, the regioselectivity with most arenes still remains problematic, unless the (hetero)arene is sterically or electronically biased. A mechanistic scrutiny of the FujiwaraMoritani reaction reveals that oxidative olefination occurs via initial electrophilic palladation. Thus, palladation is expected to occur at the more nucleophilic position of the arene guided by the innate stereo-electronic factors. However, available thermal energy to promote the reaction is sufficiently high to perturb the regio-selection as multiple $\mathrm{C}-\mathrm{H}$ bonds of arenes are prone to undergo activation, which results in multiple unwanted products formations. This genre of non-directed $\mathrm{C}-\mathrm{H}$ functionalization chemistry could herald the next phase of modern organic synthesis particularly in expanding the chemical space through late-stage functionalization; provided there is no compromise on the regioselectivity ${ }^{5-8}$. Presumably, along with the ligand assistance, a milder energy source is required which would be sufficient for the $\mathrm{C}-\mathrm{H}$ activation while allowing the desired regio-selection only through kinetically driven process. In addition, a directing group strategy can be employed in conjunction with mild condition (Fig. $1 c)^{9-13}$.

The paradigm of merging transition metal catalysis with photocatalysis has invoked mechanistic wonders in cross-coupling reactions ${ }^{14-22}$. This redefining of chemistry is forged either by a modulated 
oxidation state or in situ generation of an excited catalytic species through energy transfer ${ }^{23}$. Despite the economic and operational benefits ${ }^{24}$, its application in $\mathrm{C}$-H activation with palladium catalysis remains elusive ${ }^{25}$. Converging the appealing properties and complementary reactivities of palladium catalysis with photocatalysis ${ }^{26,27}$ has the potential of creating diverse $\mathrm{C}-\mathrm{H}$ activation manifolds in a sustainable manner, and this combination fits aptly in the current context of regioselective Fujiwara-Moritani reaction (Fig. 1d). Here, we describe how Pd/organic photocatalysis can achieve the regioselective olefination of arenes and heteroarenes (Fig. $1 \mathrm{f}$ and $1 \mathrm{~g}$ ). As per an intuitive mechanistic postulate, $\mathrm{Pd}$ /light combination serves the purpose of the $\mathrm{C}-\mathrm{H}$ activation while bypassing the thermal activation mode (Fig. 1e). Presumably, the photoexcitation of Pd species upon energy transfer in the presence of a photocatalyst and visible light source attribute to the $\mathrm{C}-\mathrm{H}$ activation process. The mildness of the light energy allows the regioselective palladation to occur at the most electron-rich site. The same photocatalyst/light merger is also effective for the regeneration of $\mathrm{Pd}(\mathrm{II})$ from $\mathrm{Pd}(0)$ to continue the cycle $\mathrm{e}^{28}$. Since the redox potential has a crucial role to play in the dual functioning of activation and oxidation, a prudent choice of organophotocatalyst is necessary. Most notably, the present catalytic system obviates the use of superstoichiometric silver salt as the oxidant, although it is known to play a pivotal role to regenerate the active catalyst in the reaction under thermal conditions ${ }^{29}$.

We initiated our investigations with the photo-induced olefination of arenes via non-directed approach. From the established redox potentials of organo-photocatalyst eosin- $Y^{30}$ and $\mathrm{Pd}(\mathrm{II}) / \mathrm{Pd}(0)$, the regeneration of the active catalyst can be conceived. However, the point of uncertainty was the feasibility of initial $\mathrm{C}-\mathrm{H}$ activation step through energy transfer excitation of the Pd species. To proceed with the validation of the photoinduced $\mathrm{C}-\mathrm{H}$ activation step, a palladium complex with the pyridone ligand (L10) was structurally characterized by X-ray crystallography (Fig. 1h). The competency of this precatalyst in effectuating $\mathrm{C}-\mathrm{H}$ activation was adjudged by the absorption experiments, which showed the maxima in the visible light region, complying with the mechanistic hypothesis (Fig. 1i). Besides, absorption studies performed at an interval of 5 min on mixing the individual components of the standard reaction without olefin also indicated the formation of a similar type of Pd species which too shows absorption in the visible light region (see Supplementary Information for details, Section 3.6. Fig. S5). To verify all these observations in an experimental set up, $o$-xylene, bearing two distinct reaction sites ( $a$ and $b$ ), was subjected to olefination employing the $\mathrm{Pd}(\mathrm{OAc})_{2}$ / eosin-Y/visible light combination at room temperature under air. The outcome of this reaction was encouraging, indicating the actual path is in line with the mechanistic postulate. The Pd-precatalyst was also found to be catalytically competent in promoting the photoinduced olefination (see Supplementary Information for details, Section 2.27.). This led to a survey of various combinations of Pd salts, photocatalysts in presence of a CFL lamp ( $23 \mathrm{~W}$ ) as the visible light source. It turned out that fluorescein rendered near explicit $b$-regioselectivity alongside high yield of the olefinated product (1). The pyridone-based ligand played a decisive role in improving regioselectivity and reactivity of arenes towards photoinduced Fujiwara-Moritani reaction. However, it may be noted here that pyridine and quinoline based ligands examined for this transformation provided inferior results pertaining to regioselectivity. An extensive study of different parameters finally accomplished a reaction condition that afforded $88 \%$ of the olefinated product (1) with $b /$ a selectivity $>25: 1$ (for optimization details see 
Supplementary Information, Section 2.7. Table S1-S8). A minimal requirement of a catalytic $\mathrm{Pd}(\mathrm{OAc})_{2}$ /ligand/ photocatalyst to perform olefination at room temperature with a household CFL lamp established an ideal stage for interrogating the generality of this process in terms of scope and regioselectivity.

The optimal conditions were then implemented to explore the scope of olefins as coupling partners of $O$ xylene (Fig. 2a). An array of diversely substituted acrylates served as effective coupling partners for this transformation giving excellent yields of the desired olefinated products and maintaining high degree of b-selectivity (2-6). The present catalytic system was amenable to olefins possessing free carboxylic acid (7), ketone (8), nitrile (9), phosphonate (10) and sulfone (11), with equivalent efficiency as acrylates. With a cyclic $a, \beta$-unsaturated ester, an allylic product was obtained in a synthetically useful yield and high $b$ regioselectivity (12). In general, it is observed that acrylic acid is often incompatible in a Fujiwara-Moritani reaction; albeit defied in present transformation. We next evaluated simple arenes, which are deemed as problematic substrates in a non-directed approach owing to the formation of regioisomeric mixture of products. An exclusive formation of a single regioisomer was observed in all cases of mono-substituted arenes irrespective of their electronic nature (Fig. 2b). While toluene (14), anisole (15) and chlorobenzene (16) provided exclusive para-selectivity; nitrobenzene (18), trifluoromethyl benzene (19) and benzaldehyde (20) gave exclusive meta-selectivity and that with fluorobenzene (21) exclusive orthoselectivity was observed. Noteworthy that even with highly electron-withdrawing effects of $-\mathrm{NO}_{2}$ and $-\mathrm{CF}_{3}$ the reactivity and regioselectivity were not hampered. The intriguing aspects continued with exclusive para-olefination of free phenol (17) obviating the prior protection protocol. A comparison of regioselectivity with these arenes under a similar thermally controlled condition establishes the superiority of the present one ${ }^{3}$. Even with disubstituted arenes having different substitution patterns (2225), regiospecific olefination occurred to provide the respective products in synthetically useful yields. The selectivity in all these arenes are expected to be primarily dictated by the electron density where palladation is favored together with the steric influence imparted by substituents and ligand. For naphthalene the maximum $b / a$ selectivity that could be attained was 14:1 utilizing 5-chloro substituted pyridone (L3) as the suitable ligand (26). The compatibility was further showcased by the regiocontrolled olefination of ferrocene derivative (27).

As with the arenes, the heteroarenes also possess multiple reaction sites (Fig. 2c). Scrutiny of literature reports unveils $\mathrm{C}-\mathrm{H}$ olefination of common five- and six-membered heterocycles such as furan, thiophene and pyridine have always led to multiple regioisomeric products ${ }^{31}$. However, in the present case the same heterocycles afforded exclusive C3-olefinated products, unattainable thus far (28-29, 34-36). With C2/C3 substituted furan and thiophene a C5-olefination was observed (30-32); regioselectivity being governed by the steric contribution of the substituent. Moreover, the unprotected imidazole was competent as substrate for the photoinduced olefination at C5 position selectively (33). Usually the presence of free $\mathrm{NH}$ group tends to impede the catalytic activity or even gives rise to aza-Michael reaction but proficient under the current system; depicting the tolerance level of the transformation. With pyrimidine, a C2 selective olefination was observed (37); the acidity of the $\mathrm{C} 2-\mathrm{H}$ aiding the regioselective palladation for this case. 
With bicyclic heteroaromatics such as quinoline (38) and indole (39), exclusive C3 olefinated products were obtained in good yields. Benzothiazole (40) under the standard reaction conditions provided C2 olefinated product while with carbazole (41) exclusive C3 selectivity was observed. The viability of acrylates and acrylic acid leaves the scope of tethering natural products into them. Indeed, a host of acrylates with complex natural product pendants viz. cholesterol (42), geraniol (43 and 51), fenchyl alcohol (44),d-tocopherol (45), oleic acid (46), g-oryzanol (47), pregnanolone (48), ergosterol (49), estrone (50) and citronellol (52) underwent facile reactions with arene and heteroarene components without any compromise in regioselectivity (Fig. 2 d). The generation of a library of structurally diverse natural products clearly demonstrates the robustness of the protocol.

Despite the inherent advantages of non-directed approach, assistance of a directing group will continue to persist as the quintessential part for obtaining complementary regioselectivity. This propelled the implementation of photoinduced ortho olefination of arenes with focus on weak coordinating DG such as acetanilide and silanol (Fig. 3a) ${ }^{32}$. Noteworthy, a combined photo-thermal approach is reported for the synthesis of indole by oxidative olefination, restricted to intramolecular approach only ${ }^{33}$. For anilide a slightly revised reaction conditions enabled the oxidative olefination to occur in a mono-selective fashion and high yields. An array of acrylates (53-55, 58), a, $\beta$-unsaturated ketones (56) and sulfones (57and 59) could be incorporated at ortho-position with the aid of photocatalysis. The transformation worked with equal efficiency for substituted acetanilides irrespective of their position and electronic nature (57-59). Even pivalamide as a DG proved efficient for ortho-olefination of naphthalene system under the present photocatalytic conditions (60 and 61). With benzyl silanol system, eosin-Y provided superior results over other organo-photocatalysts (for optimization details see Supplementary Information, Section 2.9. Table S18). The modified conditions led to high reactivity for ortho-olefination even at room temperature despite the weak coordinating nature of DG (62-64). The big lead that evolved out of the ortho strategy is the first photoinduced axial chirality at the biaryls by dynamic kinetic resolution (Fig. 3b). In presence of (L)-N-Ac-Ala as the chiral ligand and a heteroleptic iridium photocatalyst facile olefination occurred at the targeted position of the biaryl system leading to atropisomers with high degree of enantioselectivity (6566).

The merger of palladium catalysis with photocatalysis was pursued further with the aim of performing DG assisted distal meta-and para-C-H olefination; the genre which remained completely unprecedented till date. Usually it has been noticed that for a successful outcome of Pd catalyzed olefination at distal positions, the requirement of silver salt is apparent. In this aspect several computational studies have been carried out that hints towards the formation of $\mathrm{Ag}-\mathrm{Pd}$ heterobimetallic system, which is responsible for a better distal regioselectivity ${ }^{34}$. A fascinating fact observed from the present strategy is the attainment of exclusive distal selectivity at room temperature even in the absence of silver salt (Fig. 3c). In the context of meta-C-H olefination, 4-methoxy-2-cyano-phenol was utilized as the directing group with linkers such as sulfonyl (67-72), silyl (73-74), phosphonate (75-76) and carboxylate (77-84). Diverse forms of the substrates were well tolerated and provided excellent meta-selectivity with good yields of the respective olefinated products using eosin-Y as the optimal photocatalyst. The protocol was suitable in 
accommodating a range of acrylates, vinyl ketones, sulfones, phosphonates and pthalimides. Even with the extended linker length there was no drop in regioselectivity (78-80). The biphenyl carboxylic acid was also suitable as substrate in providing the desired product upon reaction with cinnamaldehyde (81). This protocol also marks the use of 2-amino benzonitrile as an effective DG for meta-olefination of phenyl acetic acid derivatives (82). Finally marketed drugs and agrochemicals were subjected to late-stage functionalization by the present catalytic system. Ibuprofen (NSAID) and clofibric acid (herbicide) were transformed to their meta-olefinated derivatives in useful selectivities (83-84). The photoinduced para-C-H olefination was next sought after using our recently developed second-generation para-DG (Fig. $3 \mathrm{~d})^{35}$. The results obtained were exceedingly satisfying; in most cases the para-selectivity was beyond 30:1 using eosin-B as the optimal photocatalyst. The substituent compatibility is considerable ranging from electron-donating to electron-withdrawing ones (85-94). To the best of our knowledge, this constitutes the first report on distal meta-and para-C-H activation by photoexcitation.

The development of benign reaction conditions for highly regioselective oxidative olefination by nondirected approach gives ample opportunity to harness its potential for the synthesis of drugs or conduct late-stage functionalization of bio-relevant molecules. Such an approach is expected to broaden the scope for encompassing molecules, which are inaccessible for functionalization by DG strategy. Exclusive para-olefination observed for free phenol prompted to venture the synthesis of phenol drugs and natural products (Fig. 4a). The newly developed photoredox system expedites the synthesis of caffeic acid (95), ferulic acid (96), p-coumaric acid (97), curcumin (100), plicatin B (101), drupanin (102), artepillin C (103) and others (98-99); synthesis of which in a directed strategy demands inclusion of multistep procedures (Fig. 1j). The late-stage functionalization of various bio-relevant molecules was next targeted (Fig. 4b). Uracil (104), caffeine (105) and estrone (106) were subjected to photoinduced olefination, all of which furnished moderate to good yields of the olefinated products in exclusive regioselectivity. [2.2]Paracyclophane, commonly utilized as a ligand, was desymmetrized through selective mono-olefination (107). Furthermore, the nonsteroidal anti-inflammatory drugs celecoxib (108), indoprofen (110), flurbiprofen (111), ketoprofen (112), ibuprofen (113) and fenbufen (114) were olefinated in synthetically useful yields and high regioselectivity, creating an expanded chemical space. $\mathrm{C}-\mathrm{H}$ Olefination of homoveratric acid (human urinary metabolite) also proceeded smoothly to give the desired product (109) in exclusive selectivity. Strychnine, known for its pesticide activity, could also be olefinated (115) in an exclusive regioselective fashion applying this protocol.

Based on our proposed design plan, the fundamental part of this metallaphotoredox transformation is the dual role of visible light as an oxidant and activator. In order to probe that, a series of control experiments were performed in a stepwise manner. In absence of either the photoredox catalyst or visible light, a minimal conversion was observed which suggests that in the oxidation step their role is imperative in regenerating the active catalyst (Fig. 4c, eqn. 1 and 2). A standard reaction was carried out with degassed solvent and liquid components under argon atmosphere that led to trace amount of the product (Fig. 4c, eqn. 3). The same reaction with $100 \mathrm{~mol} \%$ of the photoredox catalyst gave the corresponding product in $48 \%$ yield (see Supplementary Information, Section 3.1. [3.1D.]). This indicates 
$\mathrm{PC} /$ visible light merger can itself serve as the oxidant but in presence of oxygen a peroxy species generated in situ can simultaneously assist as an additional oxidant and therefore have beneficial effects on the reaction ${ }^{28}$. The visible light has an integral role to play in the $\mathrm{C}-\mathrm{H}$ activation step as well. This was adjudged from a model reaction with a meta-DG containing substrate in the absence of olefin wherein a dimerization occurred by cross-dehydrogenative coupling engaging the distal meta-C-H bonds (116) (Fig. $4 d$, eqn. 1). The same reaction in dark condition at room temperature reverted back the substrate (Fig. $4 d$, eqn. 2). Formation of selective meta dimer through oxidative coupling upon light irradiation indicates that the threshold energy required for $\mathrm{C}-\mathrm{H}$ activation step is fulfilled by photochemical energy.

In order to rationalize the excellent regioselectivity observed for the $\mathrm{C}-\mathrm{H}$ olefination of various arenes in the present transformation, we determined Fukui values for several (hetero)arenes by computing a population analysis for each arene (see Supplementary Information for details, Section 3.8.) ${ }^{36}$. As shown in Fig. 4e, the electron-density map of partial charges correlates to the observed regioselectivity of (hetero)arenes; with the most electron dense site having the highest probability for oxidative functionalization. The conversion profile of the reaction was monitored through an on/off experiment (see Supplementary Information for details, Section 3.5. Fig. S4). Suppression in the product formation when the CFL was switched off indicates the requirement of constant photo excitation for an effective transformation (see Supplementary Information for details, Section 3.5. Fig. S4). In order to analyze the mode of reaction, three independent reactions were performed with three equivalence of 2,2,6,6tetramethylpiperidin-1-oxyl (TEMPO), phenyl $N$-tert-butylnitrone (PNB) and butylated hydroxytoluene (BHT), respectively (see Supplementary Information for details, Section 3.3.). None of these reactions exhibited any noticeable impediment of catalytic activity, referring that radical reaction is unlikely to be operative. Further, a higher value of KIE for both non-directed and directed strategy implies the $\mathrm{C}-\mathrm{H}$ scission to be involved during the rate-determining step (see Supplementary Information for details, Section 3.4. Fig. $\mathrm{S} 2-\mathrm{S} 3$ ).

In summary, we have devised a palladium photoredox protocol for accessing photoinduced FujiwaraMoritani reaction by both the non-directed and directed approaches without the requirement of silver salts and thermal energy. Present transformation highlights the dual role of visible light as an oxidant and activator in enabling the following: (a) highly regioselective olefination of (hetero)arenes via non-directed approach; (b) expedite the synthetic routes to phenolic natural products; (c) photoinduced atroposelective implementation of axial chirality by using a chiral ligand; (d) first distal functionalization by photocatalysis and (e) late-stage functionalization of bio-relevant molecules. The mechanistic finding brings forward an elusive form of this transformation into light through the photoexcitation of palladium catalyst and is expected to create a paradigm for wide scale industrial and biological applications.

\section{References}

1. Moritani, I. \& Fujiwara, Y. Aromatic substitution of styrene-palladium chloride complex. Tetrahedron Lett. 8, 1119-1122 (1967). 
2. Le Bras, J. \& Muzart, J. Intermolecular dehydrogenative Heck reactions. Rev. 111, 1170-1214 (2011).

3. Wang, P., Verma, P., Xia, G., Shi, J., Qiao, J. X., Tao, S., Cheng, P. T. W., Poss, M. A., Farmer, M. E., Yeung, K.-S. \& Yu, J.-Q. Ligand-accelerated non-directed C-H functionalization of arenes. Nature 551, 489494 (2017).

4. Chen, H Wedi, P., Meyer, T., Tavakoli, G. \& van Gemmeren, M. Dual ligand-enabled non-directed C-H., olefination of arenes. Chem.

5. Kuhl, N., Hopkinson, M. N., Wencel-Delord, J. \& Glorius, F. Beyond directing groups: transition-metalcatalyzed C-H activation of simple arenes. Chem. Int. Ed. 51, 10236-10254 (2012).

6. Hartwig, J. F. \& Larsen, M. A. Undirected, homogeneous C-H bond functionalization: challenges and opportunities. ACS Cent. Sci. 2, 281-292 (2016).

7. Berger, F., Plutschack, M. B., Riegger, J., Yu, W., Speicher, S., Ho, M., Frank, N. \& Ritter, T. Site-selective and versatile aromatic $\mathrm{C}-\mathrm{H}$ functionalization by thianthrenation. Nature 567, 223-228 (2019).

8. Börgel, J. \& Ritter, T. Late-stage functionalization. Chem 6, 1877-1887 (2020).

9. Murai, S., Kakiuchi, F., Sekine, S., Tanaka, Y., Kamatani, A., Sonoda, M. \& Chatani, N. Efficient catalytic addition of aromatic carbon-hydrogen bonds to olefins. Nature 366, 529-531 (1993).

10. Leow, D., Li, G., Mei, T.-S.\& Yu, J.-Q.Activation of remote meta-C-H bond assisted by an end-on template. Nature 486, 518-522 (2012).

11. Bag, S., Patra, T., Modak,A., Deb, A., Maity, S., Dutta, U., Dey, A., Kancherla, R., Maji, A., Hazra, A., Bera, M.\& Maiti, D.Remote para-C-H functionalization of arenes by a D-shaped biphenyl template-based assembly. Am. Chem. Soc. 137, 11888-11891 (2015).

12. Chen, Z., Wang, B., Zhang, J., Yu, W., Liu, Z. \& Zhang, Y. Transition metal-catalyzed C-H bond functionalizations by the use of diverse directing groups. Chem. Front. 2, 1107-1295 (2015).

13. Dey, A., Sinha, S. K., Achar, T. K. \& Maiti, D. Accessing remote meta- and para-C(sp $\left.{ }^{2}\right)-H$ Bonds with covalently attached directing groups. Chem. Int. Ed. 58, 10820-10843 (2019).

14. Twilton, J., Le, C. C., Zhang, P., Shaw, M. H., Evans, R. W. \& MacMillan, D. W. C. The merger of transition metal and photocatalysis. Rev. Chem. 1, 0052, (2017).

15. Terrett, J. A., Cuthbertson, J. D., Shurtleff, V. W. \& MacMillan, D. W. C. Switching on elusive organometallic mechanisms with photoredox catalysis. Nature 524, 330-334 (2015).

16. Corcoran, E. B., Pirnot, M. T., Lin, S., Dreher, S. D., DiRocco, D. A., Davies, I. W., Buchwald, S. L. \& MacMillan, D. W. C. Aryl amination using ligand-free Ni(II) salts and photoredox catalysis. Science 353, 279-283 (2016).

17. Le, C., Chen, T. Q., Liang, T., Zhang, P. \& MacMillan, D. W. C. A radical approach to the copper oxidative addition problem: Trifluoromethylation of bromoarenes. Science 360, 1010-1014 (2018).

18. Zuo, Z., Cong, H., Li, W., Choi, J., Fu, G. C. \& MacMillan, D. W. C. Enantioselective decarboxylative arylation of a-amino acids via the merger of photoredox and nickel catalysis. Am. Chem. Soc. 138, 1832-1835 (2016). 
19. Huo, H., Shen, X., Wang, C., Zhang, L., Röse, P., Chen, L.-A., Harms, K., Marsch, M., Hilt, G. \& Meggers, E. Asymmetric photoredox transition-metal catalysis activated by visible light. Nature 515, 100-103 (2014).

20. Schweitzer-Chaput, B., Horwitz, M. A., de Pedro Beato, E. \& Melchiorre, P. Photochemical generation of radicals from alkyl electrophiles using a nucleophilic organic catalyst. Chem. 11, 129-135 (2019).

21. Huang, H.-M., Koy, M., Serrano, E., Pflüger, P. M., Schwarz, J. L. \& Glorius, F. Catalytic radical generation of $\pi$-allylpalladium complexes. Catal. 3, 393-400 (2020).

22. Ham, J. S., Park, B., Son, M., Roque, J. B., Jurczyk, J., Yeung, C. S., Baik, M.-H. \& Sarpong, R. C-H/C-C functionalization approach to N-fused heterocycles from saturated azacycles. Am. Chem. Soc. 142, $13041-13050$ (2020).

23. Buzzetti, L., Crisenza, G. E. M. \& Melchiorre, P. Mechanistic studies in photocatalysis. Chem. Int. Ed. 58, 3730-3747 (2019).

24. Crisenza, G. E. M. \& Melchiorre, P. Chemistry glows green with photoredox catalysis. Commun. 803, DOI: 10.1038/s41467-019-13887-8 (2020).

25. Guillemard, L. \& Wencel-Delord, J. When metal-catalyzed C-H functionalization meets visible-light photocatalysis. Beilstein J. Org. Chem. 16, 1754-1804 (2020).

26. Chuentragool, P., Kurandina, D. \& Gevorgyan, V. Catalysis by visible light photoexcited palladium complexes. Chem. Int. Ed. 58, 11586-11598 (2019).

27. Torres, G. M., Liu, Y. \& Arndtsen, B. A. A dual light-driven palladium catalyst: Breaking the barriers in carbonylation reactions. Science 368, 318-323 (2020).

28. Fabry, D. C., Zoller, J., Raja, S. \& Rueping, M. Combining rhodium and photoredox catalysis for $\mathrm{C}-\mathrm{H}$ functionalizations of arenes: Oxidative Heck reactions with visible light. Chem. Int. Ed. 53, 1022810231 (2014).

29. Bay, K. L., Yang, Y.-F. \& Houk, K. N. Multiple roles of silver salts in palladium-catalyzed C-H activations. Organomet. Chem. 864, 19-25 (2018).

30. Hari, P. D., \& König, B. Synthetic applications of eosin Y in photoredox catalysis. Commun. 50, 66886699 (2014).

31. Ye, M., Gao, G.-L. \& Yu, J.-Q. Ligand-promoted C-3 selective C-H olefination of pyridines with Pd catalysts. Am. Chem. Soc. 133, 6964-6967 (2011).

32. Wang, C \& Ge, H. Silanol as a removable directing group for the Pd"-catalyzed direct olefination of arenes. Eur. J. 17, 14371-14374 (2011).

33. Zoller, J., Fabry, D. C., Ronge, M. A. \& Rueping, M. Synthesis of indoles using visible light: photoredox catalysis for palladium-catalyzed C-H activation. Chem. Int. Ed. 53, 13264-13268 (2014).

34. Yang, Y.-F., Cheng, G.-J., Liu, P., Leow, D., Sun, T.-Y., Chen, P., Zhang, X., Yu, J.-Q., Wu, Y.-D. \& and Houk, K. N. Palladium-catalyzed meta-selective $\mathrm{C}-\mathrm{H}$ bond activation with a nitrile-containing template: Computational study on mechanism and origins of selectivity. Am. Chem. Soc. 136, 344-355 (2014). 
35. Maji, A., Guin, S., Feng, S., Dahiya, A., Singh, V. K., Liu, P. \& Maiti, D. Experimental and computational exploration of para-selective silylation with a hydrogen-bonded template. Chem. Int. Ed. 56, 1490314907 (2017).

36. Fosu, S. C., Hambira, C. M., Chen, A. D., Fuchs, J. R. \& Nagib, D. A. Site-selective C-H functionalization of (hetero)Arenes via transient, non-symmetric iodanes. Chem 5, 417-428 (2019).

\section{Methods Summary}

\section{Photoinduced non-directed C-H olefination}

In an oven-dried screw capped reaction tube was charged with magnetic stir-bar, corresponding arene (0.1 $\mathrm{mmol}), \mathrm{Pd}(\mathrm{OAc})_{2}$ (10 mol\%), 2-hydroxy-3-nitro-5-methylpyridine (20-30 mol\%), fluorescein (3 mol\%), and olefin ( $0.2 \mathrm{mmol}$ ) in $1 \mathrm{~mL}$ (for $0.1 \mathrm{mmol}$ scale) of 1,1,1,3,3,3-hexafluoro-2-propanol (HFIP) were added. The reaction tube was capped and placed $3 \mathrm{~cm}$ away from four $23 \mathrm{~W}$ house hold CFL bulbs with stirring (1500 rpm) for $28 \mathrm{~h}$ to $48 \mathrm{~h}$. The temperature was maintained at $30^{\circ} \mathrm{C}$ through cooling with a fan. Upon completion the mixture was diluted with EtOAc and filtered through a celite pad. The filtrate was evaporated under reduced pressure and the crude mixture was purified by column chromatography using silica (100-200 mesh size) and petroleum ether/ ethyl acetate as the eluent.

\section{Declarations}

\section{Acknowledgements}

Financial support received from SERB; India is gratefully acknowledged (CRG/2018/003951). Financial support received from CSIR-India (fellowship to A.S., G.P.), UGC-India (fellowship to T.B., N.G., S.K.S., C.H.B.), and IIT Bombay (S.G., W.A., S.S., A.S.S.) is gratefully acknowledged. Sanjib Panda is gratefully acknowledged for Fukui value calculation and Jyoti Prasad Biswas is gratefully acknowledged for UVvisible experiments.

\section{Author contributions}

A.S., S.G., D.M. conceived the concept. A.S., W.A., T.B., S.S., N.G., S.K.S., G.P., S.K.S., C.H.B. performed the reactions and analyzed the products. A.S., D.M. designed the control experiments and mechanistic pathway. A.S.S. performed X-ray diffraction analysis and analyzed the structure. A.S., S.G., D.M. wrote the manuscript.

Competing interests: The authors declare no competing interest(s).

\section{Additional information}

Supplementary Information is linked to the online version of the paper at www.nature.com/nature 
Reprints and permissions information is available at www.nature.com/reprints. Readers are welcome to comment on the online version of this article at www.nature.com/nature.

Correspondence and requests for materials should be addressed to D.M. (dmaiti@iitb.ac.in).

\section{Figures}

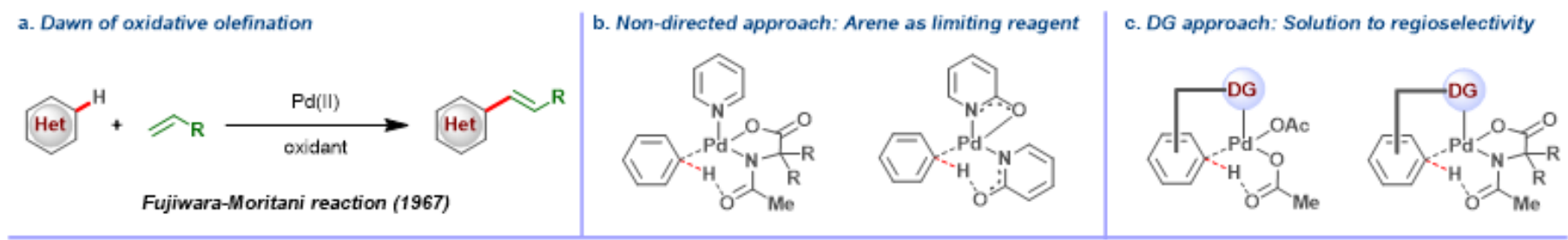

d. Towards regio-resolved Fujiwara Moritani reaction: governing factors
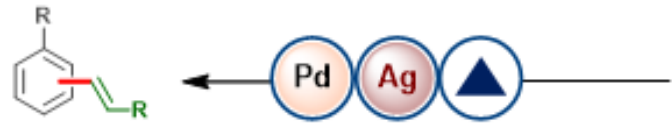

$1+\gamma_{R}^{R}$
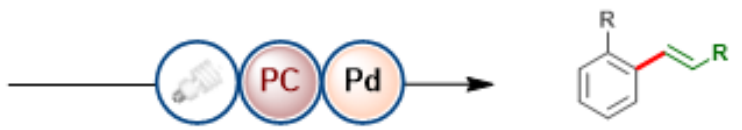

mixture of regioisomers

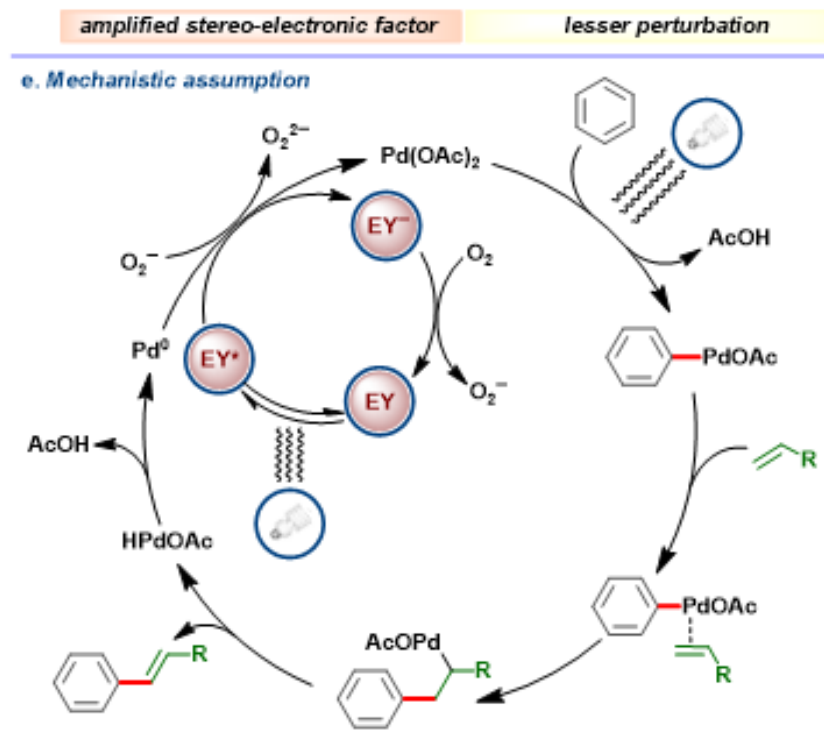

kinetically driven non-requirement of silver

single regioisomer

f. Comparative study
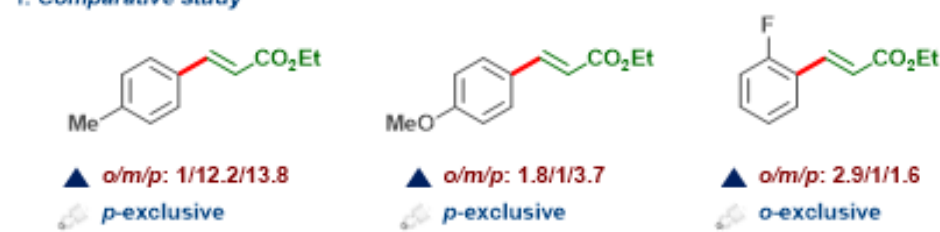

A $o / m / p: 2.9 / 1 / 1.6$

g. C3 selective olefination

Previous report

o-exclusive

H

$\mathrm{C} 2 / \mathrm{C} 3$ selective

This report

O

${ }_{S}^{H}$

C2/C3 selective

C3 exclusive

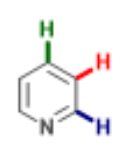

h. Crystal structure of Pd-precatalyst
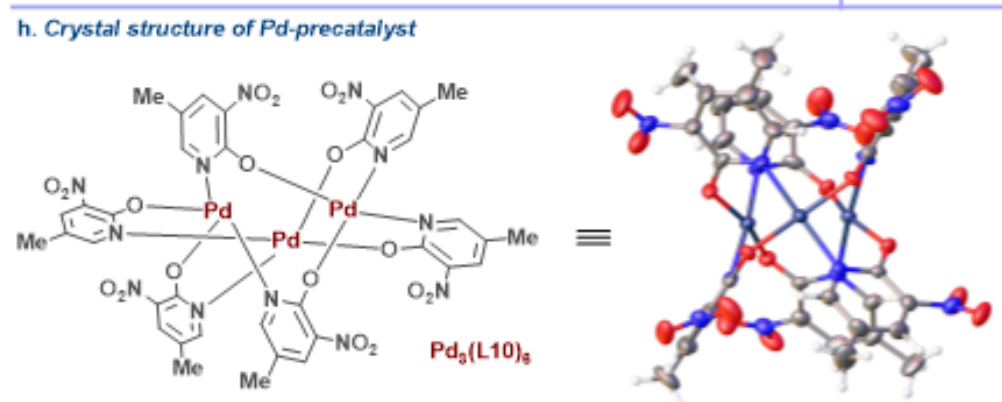

CCDC 2045974

$\mathrm{C} 2 / \mathrm{C} 3 / \mathrm{C} 4$ selective $\quad \mathrm{C} 3$ exclusive

j. This work vs DG approach: Short route to phenolic drugs
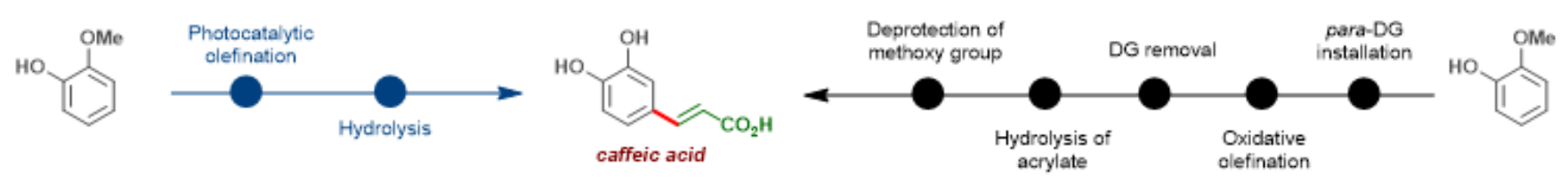


\section{Figure 1}

Evolution of Fujiwara-Moritani reaction. a, Traditional approach for oxidative olefination. b, Directing group assisted proximal and distal $\mathrm{C} \square \mathrm{H}$ activation. c, Ligand enabled Fujiwara-Moritani reaction. d, Photoinduced vs thermal Fujiwara Moritani reaction. e, Mechanistic hypothesis for the photoinduced Fujiwara-Moritani reaction. $\mathrm{f}, \mathrm{A}$ comparative study of regioselectivity for selected arene substrates under thermal and photoredox conditions. g, A comparative study of regioselectivity for selected arene substrates under thermal and photoredox conditions. h, Crystal structure of Pd-precatalyst. i, UV absorption studies of reaction components. j, A comparison of steps required to synthesize a phenolic drug via photoinduced Fujiwara-Moritani reaction in a non-directed strategy vs the directing group strategy. 

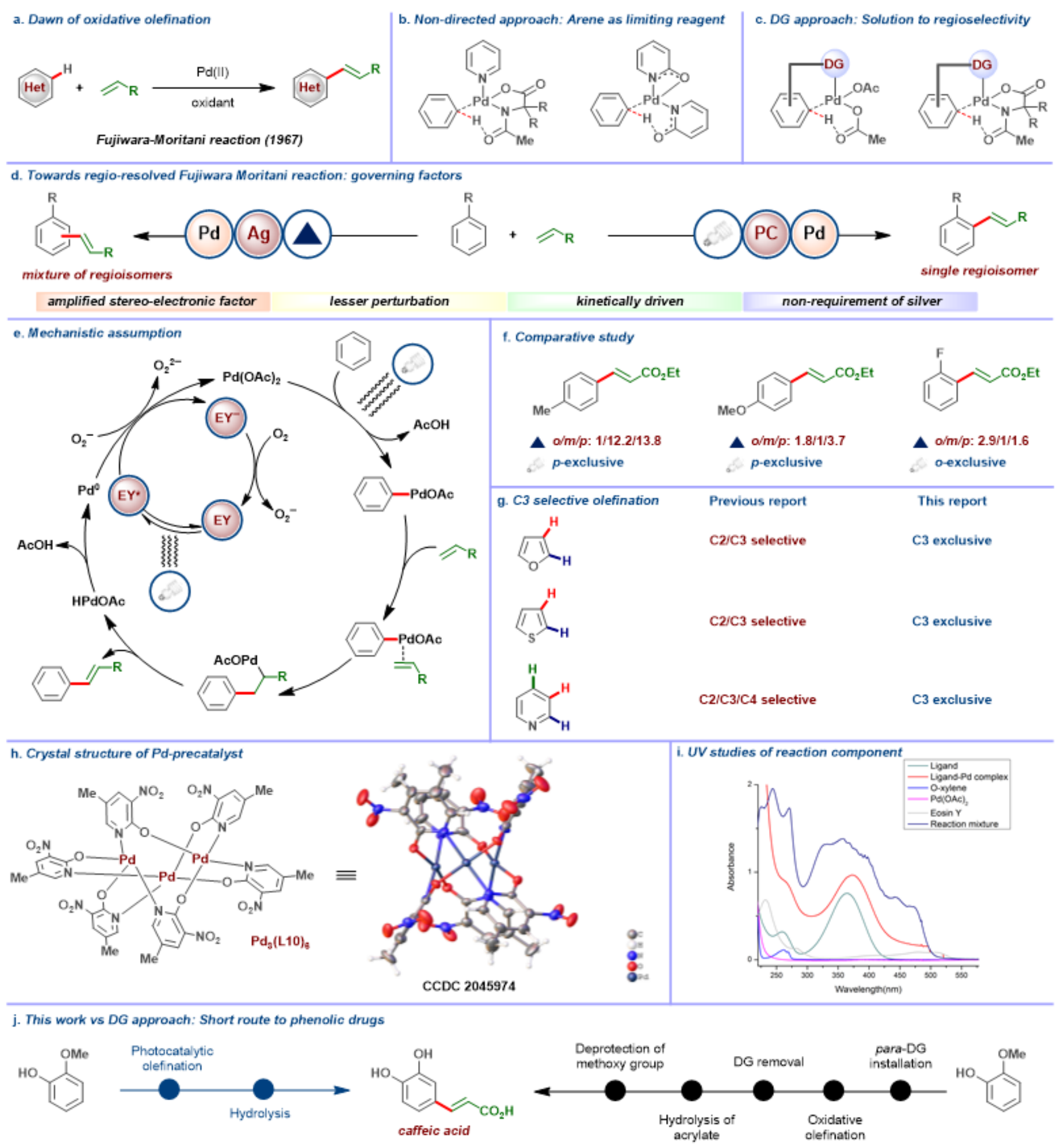

\section{Figure 1}

Evolution of Fujiwara-Moritani reaction. a, Traditional approach for oxidative olefination. b, Directing group assisted proximal and distal $\mathrm{C} \square \mathrm{H}$ activation. c, Ligand enabled Fujiwara-Moritani reaction. d, Photoinduced vs thermal Fujiwara Moritani reaction. e, Mechanistic hypothesis for the photoinduced Fujiwara-Moritani reaction. $\mathrm{f}, \mathrm{A}$ comparative study of regioselectivity for selected arene substrates under thermal and photoredox conditions. g, A comparative study of regioselectivity for selected arene 
substrates under thermal and photoredox conditions. h, Crystal structure of Pd-precatalyst. i, UV absorption studies of reaction components. j, A comparison of steps required to synthesize a phenolic drug via photoinduced Fujiwara-Moritani reaction in a non-directed strategy vs the directing group strategy.

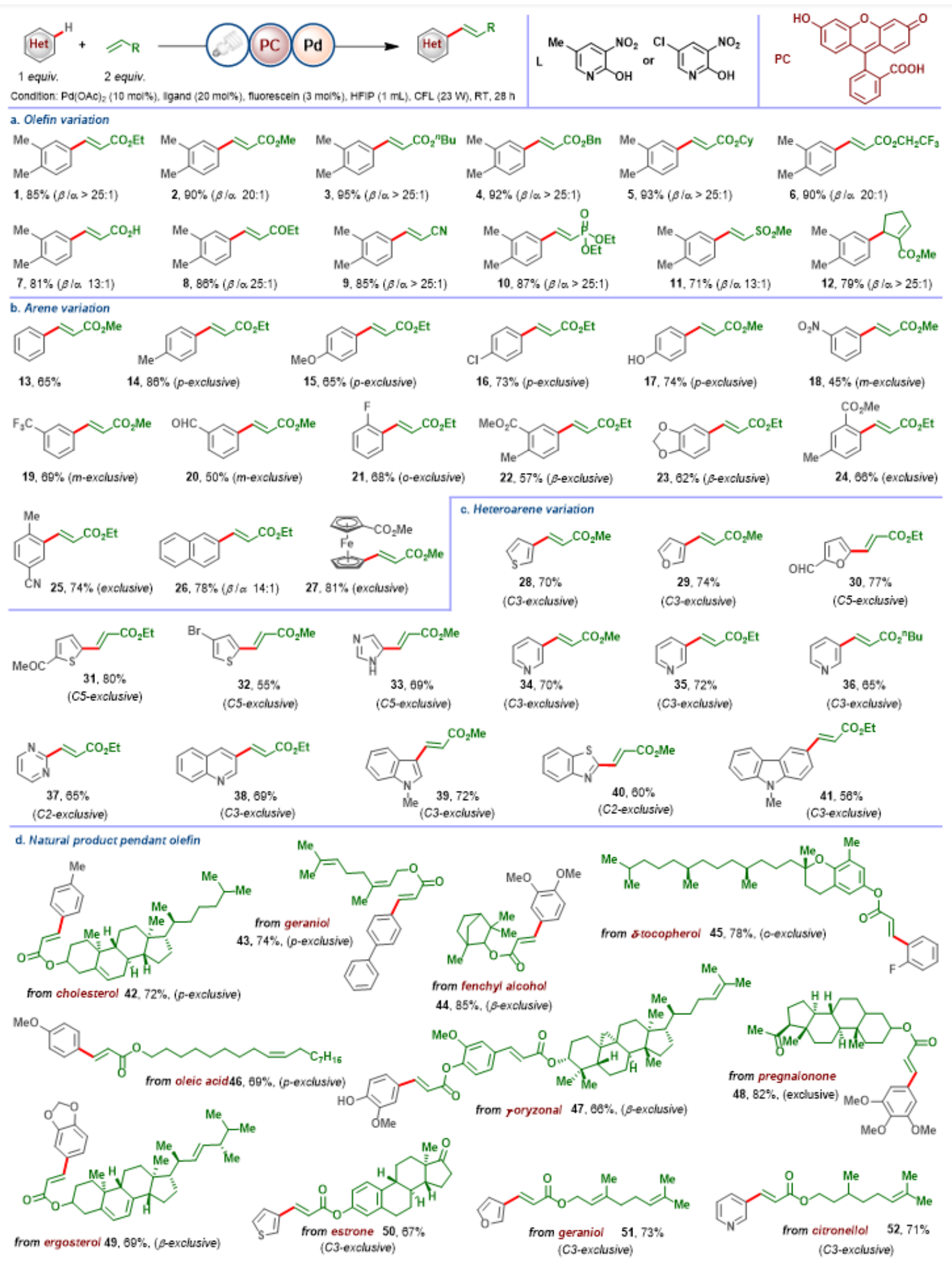

Figure 2 
Photoinduced olefination by non-directed approach. a, Scope of olefins for photoinduced olefination of oxylene. b, Scope of mono- and di-substituted arenes. c, Scope of heteroarenes towards photoinduced olefination. d, Scope of acrylates with natural product pendants. Isolated yields are indicated below each entry. See Supplementary Information for experimental details.

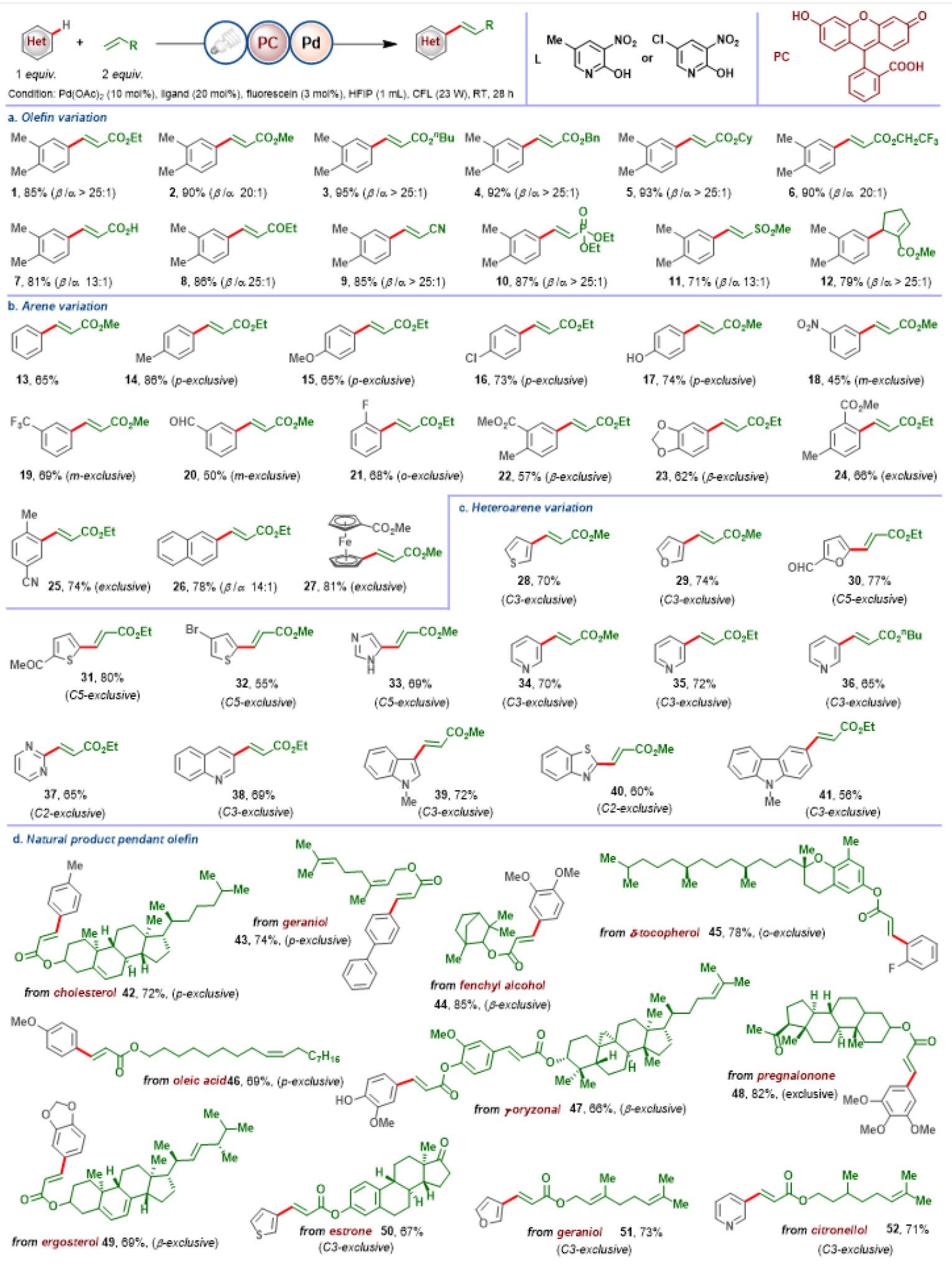

Figure 2 
Photoinduced olefination by non-directed approach. a, Scope of olefins for photoinduced olefination of oxylene. b, Scope of mono- and di-substituted arenes. c, Scope of heteroarenes towards photoinduced olefination. d, Scope of acrylates with natural product pendants. Isolated yields are indicated below each entry. See Supplementary Information for experimental details.

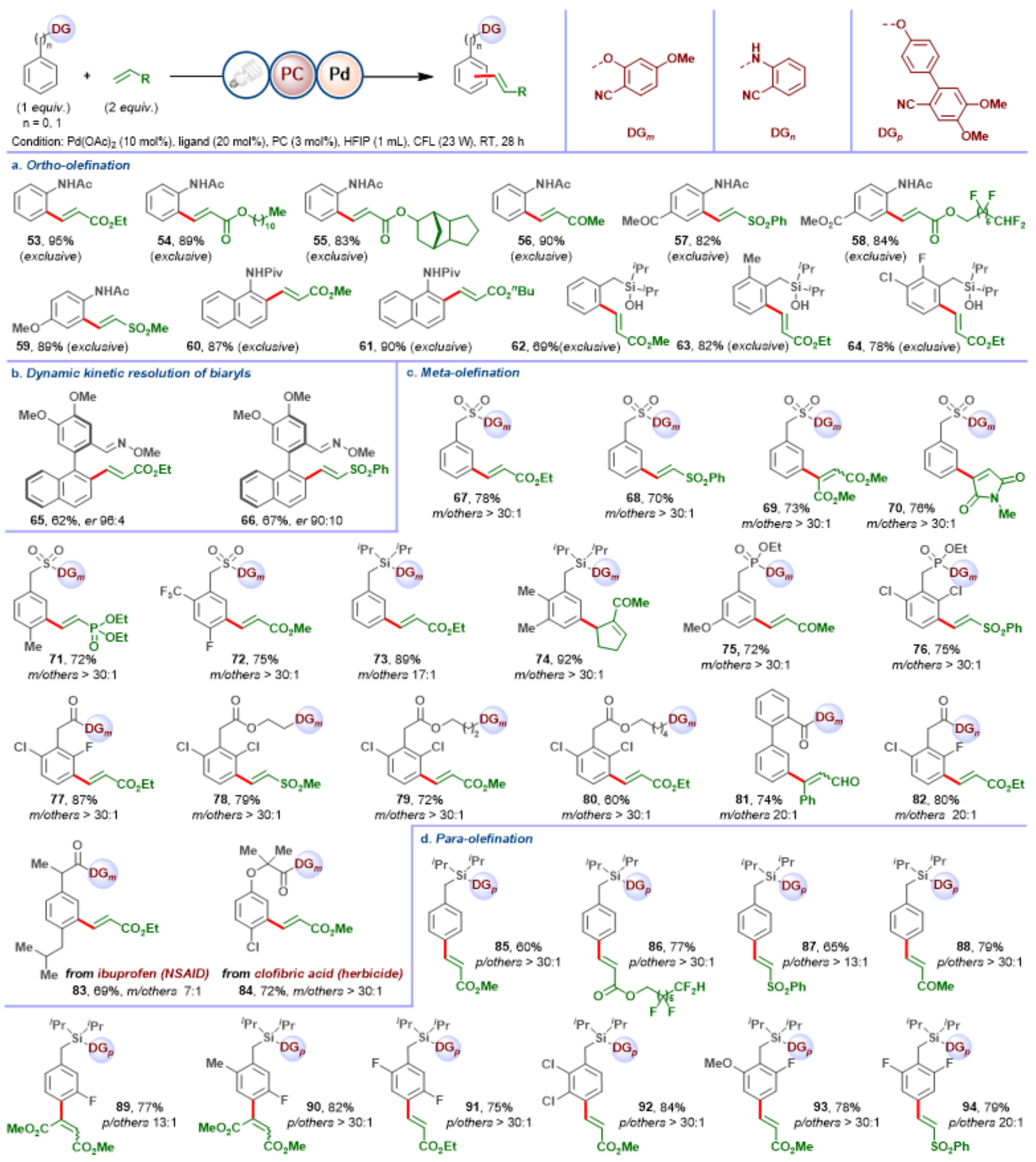

Figure 3 
Photoinduced olefination by directing group strategy. a, Ortho-olefination of anilides and benzyl silanols. b, Dynamic kinetic resolution of biaryls by photoinduced olefination. c, Photoinduced meta-olefination of arenes. The isolated yields are reported and ratios of meta:others are shown. d, Photoinduced paraolefination of arenes. The isolated yields are reported and ratios of para:others are shown. See Supplementary Information for experimental details.
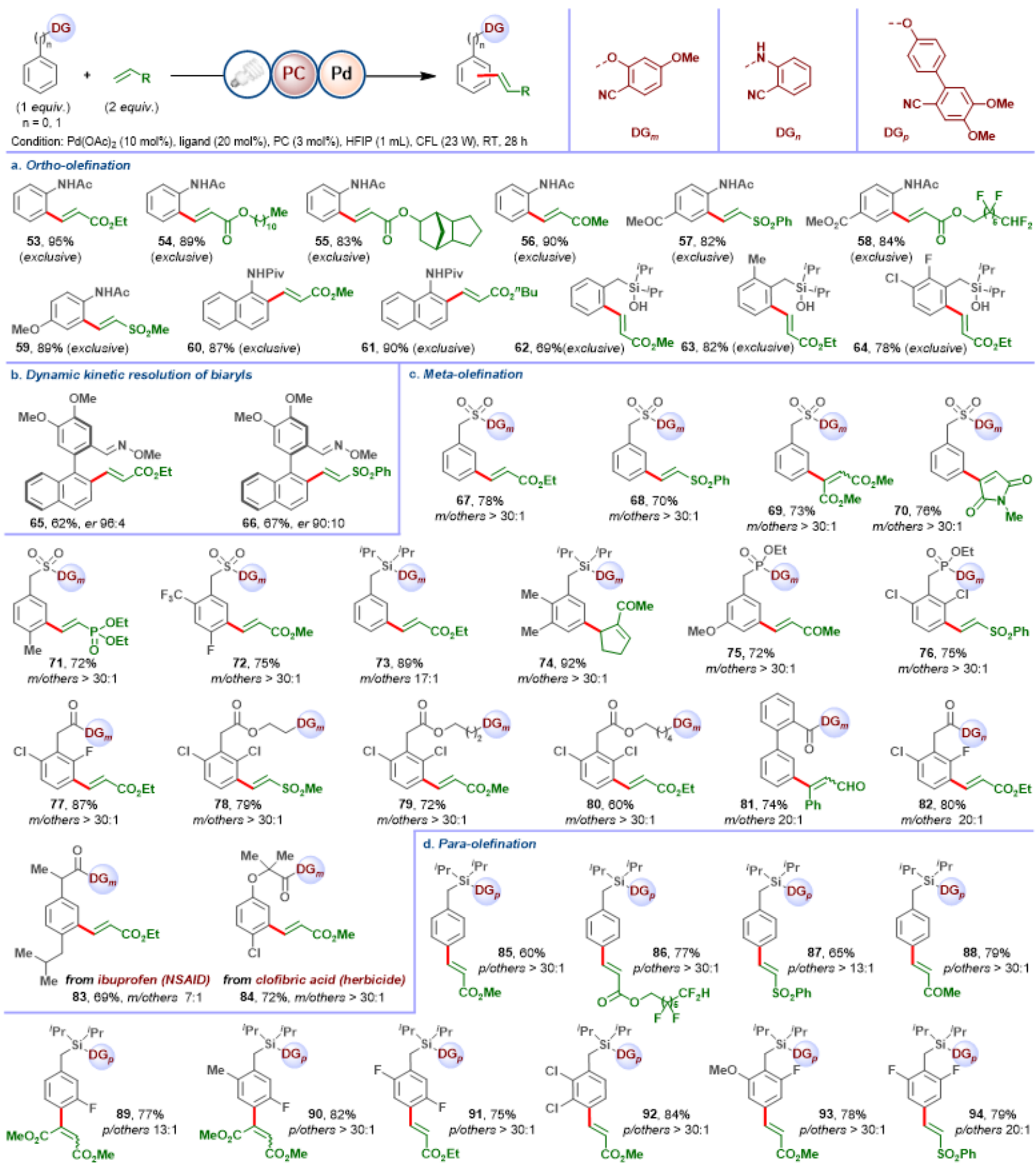

Figure 3 
Photoinduced olefination by directing group strategy. a, Ortho-olefination of anilides and benzyl silanols. b, Dynamic kinetic resolution of biaryls by photoinduced olefination. c, Photoinduced meta-olefination of arenes. The isolated yields are reported and ratios of meta:others are shown. $d$, Photoinduced paraolefination of arenes. The isolated yields are reported and ratios of para:others are shown. See Supplementary Information for experimental details.

a. Short step synthesis of phenolic drugs<smiles>CC(C)(C)C(=O)/C=C/c1ccc(O)c(O)c1</smiles>

caffeic acid: antioxidant (exclusive)

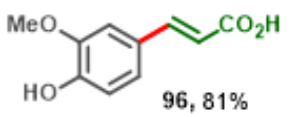

ferulic acid: antioxidant (exclusive)

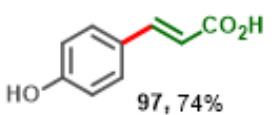

p-coumaric acid: antioxidant (p-exclusive)

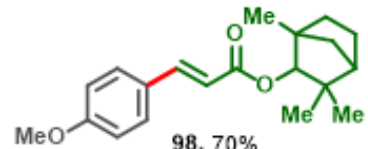

fenchyl p-hydroxycinnamate-OMe medicinal herb component (p-exclusive)

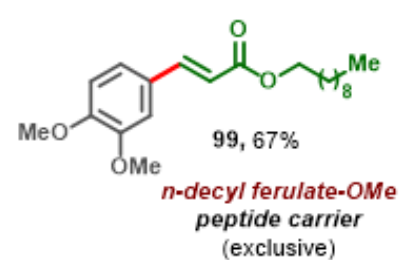
(exclusive)<smiles>COc1cc(/C=C/C(=O)CC(=O)/C=C/c2ccc(O)c(OC)c2)ccc1O</smiles>

$100,67 \%$

lammatory and antioxidant

curcumin: anti-inflammatory
(exclusive)<smiles>COC(=O)/C=C/c1ccc(O)c(CC=C(C)C)c1</smiles>

101, $62 \%$ (p-exclusive) plicatin B<smiles>CC(C)=CCc1cc(/C=C/C(=O)O)ccc1O</smiles>

$102,58 \%$ (p-exclusive) drupanin

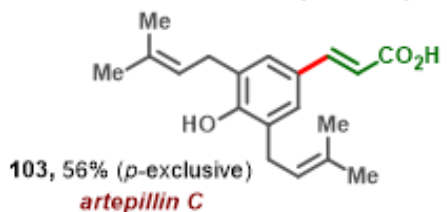

b. Late-stage functionalization of bio-relevant molecules

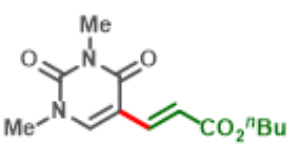

from uracil

$104,79 \%$ (exclusive)

from homoveratric acid from homoveratric acid

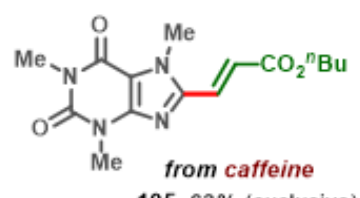
$105,63 \%$ (exclusive

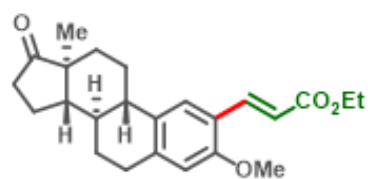

from estrone 106, 53\% 20:1

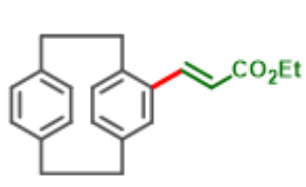

from [2.2]paracyclophane $107,42 \%$ (exclusive)<smiles>CCOC(=O)/C=C/c1cc(-n2nc(C(F)(F)F)cc2-c2ccc(C)cc2)ccc1S(=O)(=O)N(C)C(C)(C)C(C)(F)F</smiles><smiles>CCOC(=O)C=Cc1cc(N2Cc3ccccc3C2=O)ccc1C(C)C(=O)OCC(C)=O</smiles><smiles>CCOC(=O)C=Cc1cc(-c2ccccc2)c(F)cc1C(C)C(=O)OC</smiles>

$111,62 \%, 5: 1$

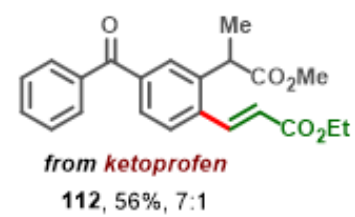

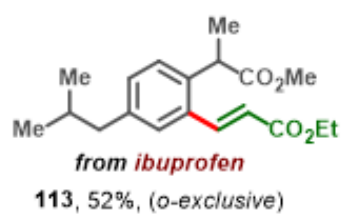

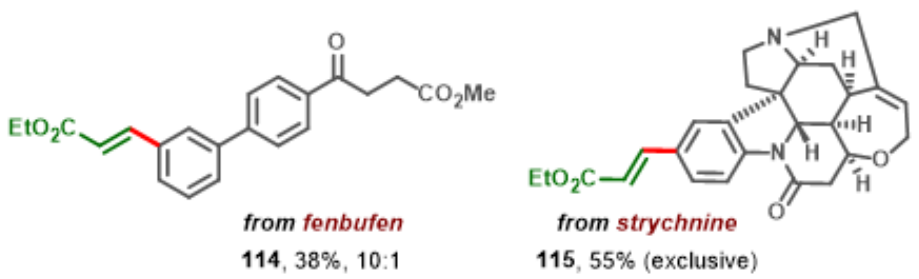

c. Role of visible light in oxidation

$\mathrm{Cl}^{\mathrm{H}}+\mathrm{CO}_{2} \mathrm{Et}$<smiles>Cc1cccc2ccccc12</smiles>

PC

under air
Pd

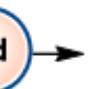<smiles>CCOC(=O)/C=C/c1ccc(C)c(C)c1</smiles>

1, $7 \%$
M

d. Role of visible light in activation
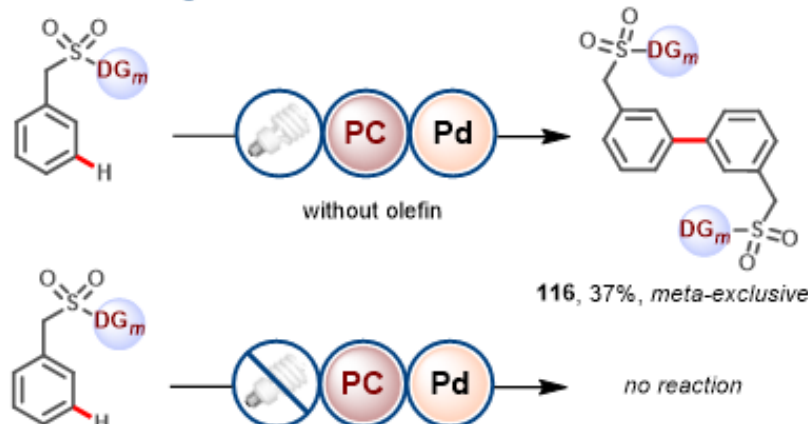

$116,37 \%$, meta-exciusive

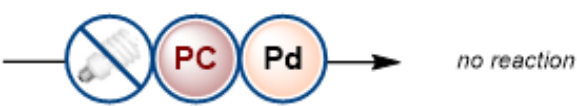

without olefin
$\overbrace{\mathrm{Me}}^{\mathrm{Me}}+\mathrm{C}_{\mathrm{CO}_{2} \mathrm{Et}}^{\mathrm{H}}$<smiles>Cc1ccccc1</smiles><smiles>CCOC(=O)C=Cc1ccc(C)c(C)c1</smiles>

under air

1, $9 \%$<smiles>CCOC(=O)C=C[CH+]c1ccc(C)c(C)c1</smiles>

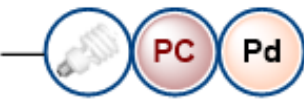

under argon

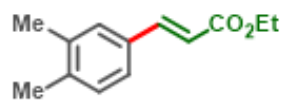

$1,4 \%$ e. Fukui value of different (hetero)arenes

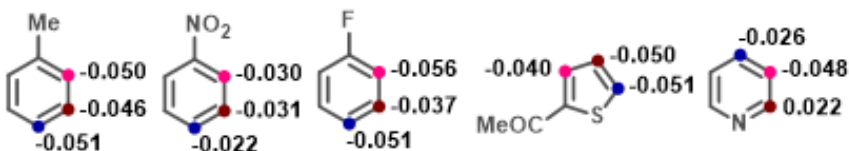

\section{Figure 4}


Applications and mechanistic investigations. a, Short-step synthesis of phenolic drugs and natural products. b, Late-stage functionalizations of bio-relevant molecules. c, Role of light in oxidation step. d, Role of light in $\mathrm{C} \square \mathrm{H}$ activation. e, Fukui values of different (hetero)arenes.

a. Short step synthesis of phenolic drugs

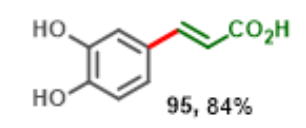

caffeic acid: antioxidant (exclusive)<smiles>COc1cc(/C=C/C(=O)O)ccc1O</smiles>

ferulic acid: antioxidant (exclusive)

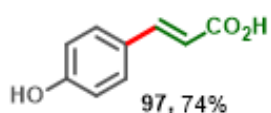

p-coumaric acid: antioxidant (p-exclusive)<smiles>COc1ccc(/C=C/C(=O)OC2C3CCC(C)(C3)C2(C)C)cc1</smiles>

fenchyl p-hydroxycinnamate-OMe medicinal herb component (p-exclusive)

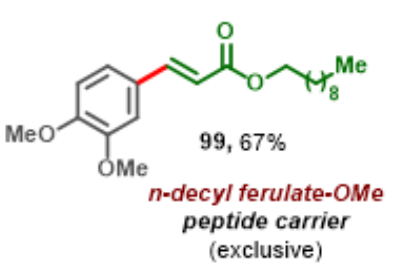
(exclusive)<smiles>COc1cc(/C=C/C(=O)CC(=O)/C=C/c2ccc(O)c(OC)c2)ccc1O</smiles>

$100,67 \%$

curcumin: anti-inflammatory and antioxidant (exclusive)<smiles>CC(=O)C=Cc1ccc(O)c(CC=C(C)C)c1</smiles>

101, $62 \%$ (p-exclusive) plicatin B<smiles>CC(C)=CCc1cc(C=CC(=O)O)ccc1O</smiles>

$102,58 \%$ (p-exclusive) drupanin

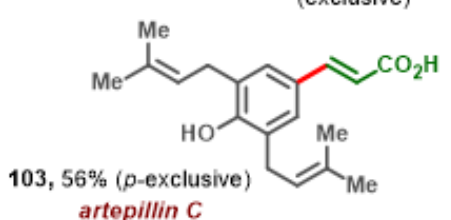

b. Late-stage functionalization of bio-relevant molecules<smiles>Cn1cc(/C=C/C(=O)OC(C)(C)C)c(=O)n(C)c1=O</smiles>

from uracil $104,79 \%$ (exclusive)

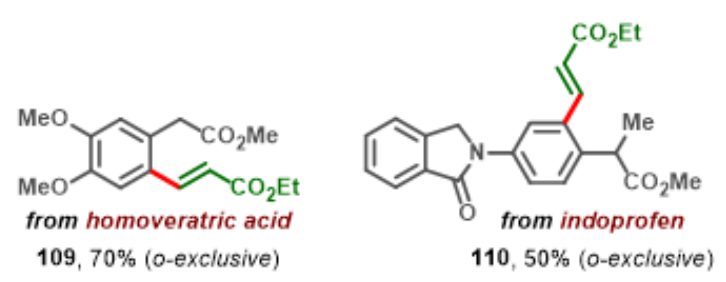<smiles>CCOC(=O)/C=C/c1cc2c(cc1OC)CCC1[C@@H]2CC[C@]2(C)C(=O)CC[C@@H]12</smiles>

from estrone $106,53 \% 20: 1$<smiles>CCOC(C)=CCc1ccccc1CCc1ccc2c(c1)CCC2</smiles>

from [2.2]paracyclophane $107,42 \%$ (exclusive)

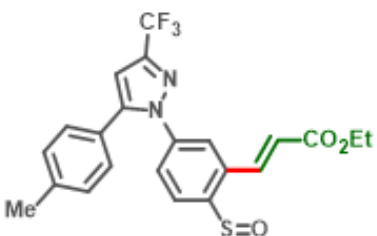

from celecoxib $\mathrm{Me}_{2} \mathrm{~N}^{\prime} \mathrm{O}$

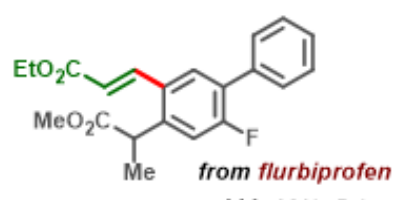

$111,62 \%, 5: 1$

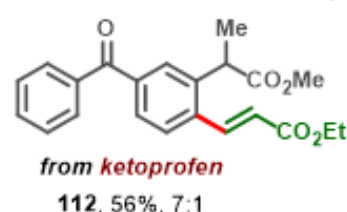

$112,56 \%, 7: 1$

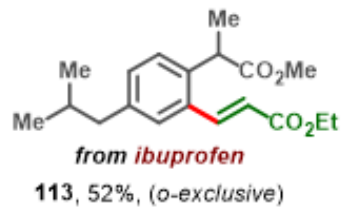

d. Role of visible light in activation<smiles>O=S(=O)([O-])Cc1ccccc1</smiles>

$115,55 \%$ (exclusive)

$$
\text { . }
$$
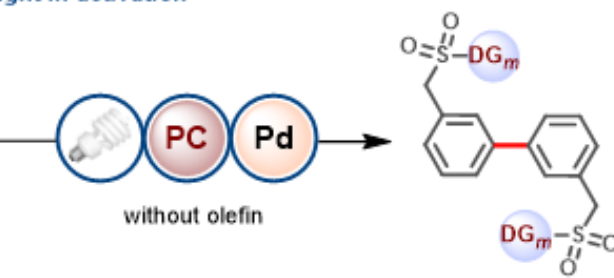

116, $37 \%$, meta-exclusive
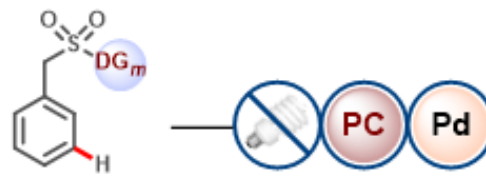

without olefin<smiles>CCOC(=O)C=Cc1ccc(C)c(C)c1</smiles>

e. Fukui value of different (hetero)arenes

$1,9 \%$<smiles>CCOC(=O)C=C[CH+]c1ccc(C)c(C)c1</smiles><smiles></smiles>

\section{Figure 4}

Applications and mechanistic investigations. a, Short-step synthesis of phenolic drugs and natural products. b, Late-stage functionalizations of bio-relevant molecules. c, Role of light in oxidation step. d, 
Role of light in $\mathrm{C} \square \mathrm{H}$ activation. e, Fukui values of different (hetero)arenes.

\section{Supplementary Files}

This is a list of supplementary files associated with this preprint. Click to download.

- SupplementaryInformationNature.docx

- SupplementaryInformationNature.docx

- checkcifPDF.pdf

- checkcifPDF.pdf

- DMAJ237.cif

- DMAJ237.cif 